Running head: DYNAMIC MODEL FOR LIMB SELECTION

\title{
A Dynamic Model for Limb Selection
}

\author{
Ralf F. A. Cox
}

Department of Psychology, University of Groningen

Correspondence to:

Grote Kruisstraat 2/1, 9712 TS Groningen, The Netherlands

(T) +31 (0)50-3636547

(E) r.f.a.cox@ @rug.nl 


\begin{abstract}
Two experiments and a model on limb selection are reported. In Experiment 1 left-handed and right-handed participants $(N=36)$ repeatedly used one hand for grasping a small cube. After a clear switch in the cube's location, perseverative limb selection was revealed in both handedness groups. In Experiment 2 the cubes were presented in a clockwise and counterclockwise sequence to right-handed participant $(N=15)$. A spatial delay in the switch point between right-hand use and left-hand use was observed. The model simulates the experiments, by implementing the multiple-timescale dynamics of the action-selection process underlying limb selection. It integrates two mechanisms that were earlier proposed to underlie this selection aspect of manual activity: limb dominance and attentional information. Finally, the model is used to simulate Gabbard et al.'s (1997) experiment, offering a concise coupling of strength and direction of handedness.
\end{abstract}

Keywords: Limb Selection, Handedness, Dynamic Model, Perseveration, Hysteresis 


\section{A DYNAMIC MODEL OF LIMB SELECTION}

Choices about which (bodily) means to use to reach a certain goal constitute an integral part of action planning in any type of daily activity, from selecting the right tool for a job to choosing the appropriate hand for grasping a coffee mug. In general, planning in goal-directed behavior entails choices about goals, means and the specifics of how to couple and sequence those goals and means. The present study addresses the action planning in limb selection, more specifically, the action-selection process underlying the choice of which hand to use as a means in performing a unimanual grasping task. The options available in such a task are of course discrete; either the right hand or the left hand, making it a bi-stable system. One important factor involved is a person's handedness, but cultural, habitual, and perceptual factors as well as task complexity contribute too (e.g. Bryden, 1999; De Agostini, Khamis, Ahui \& Dellatolas, 1997; Fagard \& Dahmen, 2003; Leconte \& Fagard, 2005; Peters, 1990; Steenhuisen \& Bryden, 1999), which makes it particular interesting as a case study for planning. Moreover, depending on the task and context, these factors can be reinforcing or competing in their contribution to the choice, temporarily favoring one hand above the other. This is evidently true for everyone who has ever experienced the difficulty of retrieving one's keys out of one's trouser pocket while holding a large bag of groceries in each hand.

As mentioned, an important controlling factor for limb selection in a unimanual task is handedness. Handedness is often considered an invariant trait that determines a person's hand use in a large number of tasks (for an overview on handedness see Hopkins \& Rönnqvist, 1998). A more contemporary view, which we will adopt here, takes other factors (i.e., from the environment and the task) into account, conceiving handedness, both in its direction and strength, as merely an internal predisposition or tendency to favor one hand above the other, not as the sole determinant in limb selection. This tendency can be counteracted when external stimulation becomes laterally specified or more demanding on the action system, as 
for example in grasping a tool in hemispace. This view is motivated by the observation that it is virtually meaningless to talk about a person's dominant limb without doing a thorough analysis of the task with which it is measured. Handedness is neither a fixed nor a static biological trait, but is highly dependent on many contextual aspects of the task. Note that from this definition, it is reasonable to view handedness as a likelihood function for using one of the hands.

As a general framework, one can roughly classify the relevant factors in limb selection into three categories of constraints, according to their relation to the action system: organismic, environmental, and task constraints (Newell, 1986, 1989; see also Clark, 1997). Each of these constraints does not serve as a single cause of behavior, but rather as a limiting factor on the action possibilities that are available for an organism (actor). The organismic constraints are best viewed as internal to the actor's action system. It means that the actor is subjected to constraints resulting from the specific biomechanics and neuro-anatomy of his or her body, but also from the history of prior actions, as far as they altered the state of the action system in any way. Environmental constraints and task constraints are external to the actor's action system and entail perceptual information with respect to the environment and the task. These constraints are placed upon the actor by the environment in which the activities are embedded, and the nature and specifics of the task at hand (i.e., its goals, the available means, but also rules, conventions, and culture). Note that the distinction between environmental constraints and task constraint is not very strict. It depends on the actor's freedom of manipulating these constraints. Note also that, in addition to their relation to the action system, another relevant and differentiating aspect of these action constraints is the timescale on which they are exerting their influence. This aspect will prove to be fundamental for our account of limb selection, and we will elaborate on it shortly. 
How do the different constraints coalesce in the planning? And how does limb selection come about as a result? In order to shed more light on these questions, Gabbard, Iteya, and Rabb (1997) performed the following simple but elegant experiment. Adult participants were asked to grasp a small object that was randomly placed at nine different locations in hemispace. The locations varied in laterality from left to right, keeping an equal distance to the body center on a half-circle in front of the participant (see Figure 1). Table 1 presents the results of their experiment. The overall response profile can be characterized as a tendency for ipsilateral reaching (i.e., the hand at the same side as the object) in the two hemispace sides, and use of the dominant limb at midline. This means that participants used their non-preferred hand when the object was presented on their non-preferred side. Although this general pattern was similar for both laterality groups, right-handers demonstrated a stronger preference for their dominant limb compared to left-handers. This can be gathered from the larger deviations from ipsilateral reaching at the non-preferred side in this group (see Table 1). Others reported comparable findings, in adults (Bryden, Pryde \& Roy, 1999, 2000; Calvert \& Bishop, 1998; Harris \& Carlson, 1993), and also in children (Gabbard, Rabb Helbig \& Gentry, 2001; Harris \& Carlson, 1993).

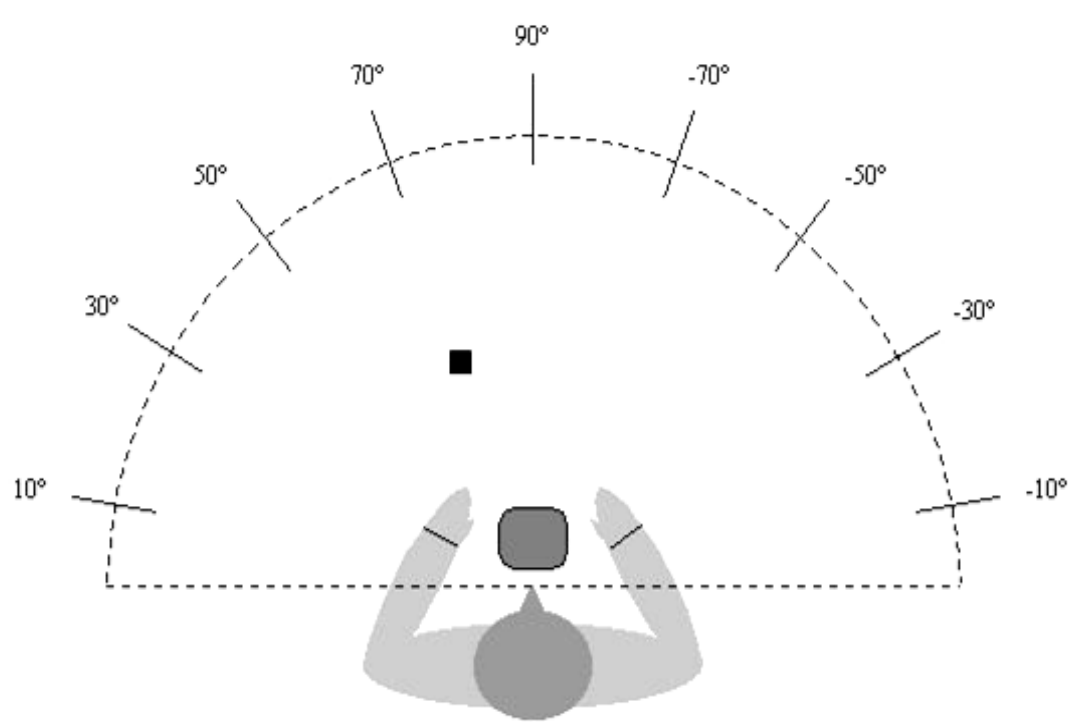

Figure 1. Setup of Gabbard et al.’s (1997) experiment. 
Table 1. Results of Gabbard et al. (1997): Proportion of preferred-hand use for the nine (randomly presented) object positions.

\begin{tabular}{cccccccccc}
\hline \multicolumn{1}{c}{ Group } & \multicolumn{3}{c}{ Left hemispace } & \multicolumn{4}{c}{ Right hemispace } \\
& $10^{\circ}$ & $30^{\circ}$ & $50^{\circ}$ & $70^{\circ}$ & $90^{\circ}$ & $-70^{\circ}$ & $-50^{\circ}$ & $-30^{\circ}$ & $-10^{\circ}$ \\
\hline Left-handers $(N=60)$ & .98 & .98 & .98 & .93 & .75 & .30 & .13 & .07 & .08 \\
Right-handers $(N=84)$ & .20 & .19 & .25 & .42 & .95 & .99 & .99 & 1.00 & 1.00 \\
\hline
\end{tabular}

Inspired by these results Gabbard and Rabb (2000; 2004) hypothesized two mechanisms underlying limb selection: limb dominance and attentional information. As stated by the authors, limb dominance, or handedness as it is often referred to, is largely responsible for the hand choice in the ipsilateral hemispace and on midline. In terms of Newell's (1986) categories of constraints, this mechanism belongs to the organismic constraints, as it is related to an asymmetry of the organism, be it functional or possibly even structural. Attentional information is supposed to be responsible for altering the hand choice in the contralateral hemispace. Gabbard and Rabb conceive the nature of the attentional information as perceptual, and its composition is determined by the spatial layout of the relevant objects in the task. Depending on the specifics of the task, it either fits into Newell's category of environmental constraints or task constraints, as it is external to the organism's action system.

Despite the obvious relevance of these mechanisms and the ample empirical evidence that stresses the multi-causality of limb selection, we still lack a general framework for integrating these ideas in a concise explanation of how the choice to use a particular hand comes about. Put differently, as to date there is no working model for limb selection that can reproduce the empirical findings. As a minor point, the mechanisms do not offer a (testable) explanation for the differences in laterality strength between left-handers and right-handers. Recently, Cox and Smitsman (2006), following Thelen, Schöner, Scheier and Smith (2001) among others (Erlhagen \& Schöner, 2002; Leconte \& Fagard, 2004; Thelen \& Smith, 
1994; see also Busemeyer \& Diederich, 2002; Busemeyer \& Townsend, 1993; Grossberg, 1980), proposed a view on action planning that conceives of planning as a dynamical actionselection process for actions to take (see also Cox \& Smitsman, 2008; Smitsman \& Cox, 2008). This view differs from the static symbol-manipulation and all-or-none type of views on planning in a number of fundamental points, which we will briefly describe below. First, choices for action are multi-causal, which means that many factors on several timescales can (potentially) influence the choice that will be made. These factors can be of very different origin and nature. Second, the process is embodied and embedded (situated) in its nature. This means that action selection, at its most fundamental level, is organized by and deals with structural and functional characteristics of the actor's body and environment. These include preferences of a functional, biomechanical, or neuro-anatomical nature. Third, the process has an intrinsic dynamics that incorporates these properties and determines the fate of the relevant factors in the process, in accordance with the neuro-anatomical structure of the action system. This makes it a continuous dynamical system in which choices for action emerge gradually over time. A final distinctive property, although not the topic of this paper, is that the process is also continuous in the sense that it does not halt after a single choice is made. Rather the dynamics of the process continues to generate subsequent choices for action, fed by the available input that may have changed. Choices are not ready made, waiting to be executed, but emerge from and remain part of the dynamics of the process. In this paper we will build on the view of planning described above to model limb selection.

One of the most elaborate models embracing a highly similar view is by Thelen et al. (2001), concerning perseverative reaching behavior in infants, better known as the A-not-B error (Piaget, 1954). Their model is based on dynamic field theory of movement preparation (for an overview see Erlhagen \& Schöner, 2002). A key aspect of Thelen et al.'s model is that the planning of a reaching movement is a process that takes place on different timescales. In 
the A-not-B task, there is the within-trial timescale of the evolution of a motor plan for a single reaching movement towards a certain location. This plan is influenced by various types of information (task and specific inputs) that are available in real time, that is, before reaching onset during the actual planning of the reaching movement. In addition to this, an over-trials timescale of a series of reaching movements is suggested. At this timescale a motor memory evolves, which builds up a short-term bias towards a particular location, each time a reach is executed in the direction of that location. In other words, shaped by these earlier reaching movements, the motor memory gradually builds over time. In the authors' perspective this motor memory influences subsequent plans for reaching movements.

A most striking example from which it becomes apparent that these timescales interact is the phenomenon of perseveration, which is at the heart of the A-not-B error. Basically, perseveration demonstrates that a choice for action at a certain point in time is affected by previous choices for actions, such that these temporarily overrule the perceptual information that is available. In the A-not-B error it manifests itself by infant's perseverance in reaching towards location A, after a number of successful reaches to that location, even after a perceptual cue is given in favor of another location (i.e., location B). Perseveration as a more general cognitive or behavioral phenomena, revealing the multiple-timescale dynamics of planning, has not had a lot of attention from researchers in the behavioural sciences. There are some studies on children's search behavior (Sophian \& Wellman, 1983), sorting (Zelazo \& Reznick, 1991), and word-naming and categorization (Deák \& Narasimham, 2003; Deák, Ray, \& Brenneman, 2003). To our knowledge there is no study reporting perseverative limb selection in adults.

The multiple-timescale dynamics reveals itself in yet another subtle way. Consider a (bi-stable) system for which two distinct modes of behavior are possible. Moreover, consider this system to be performing under a gradually or sequentially changing parameterized 
condition. When this condition changes between two extremes, for which one of the behavioral modes is most suitable (and probable), a switch between the two modes of the system is observed at some point. Fundamentally, this switch can take place at a different value of the parameter, depending on the direction of change. This effect refers to the wellknown hysteresis (and enhanced contrast) phenomenon, which is amongst the basic 'flags' of non-linear behavior in dynamical systems. Examples of hysteresis are numerous in human behavior (e.g., Farrell,1999; Haken, Kelso \& Bunz, 1985; Hock, Schöner \& Kelso, 1993; Kelso, 1995; Schöner \& Kelso, 1988; Tuller, Case, Ding \& Kelso, 1994). To our knowledge, hysteresis in limb selection has never been studied, although a task designed by Bryden, Singh, Steenhuisen, and Clarkson (1994) is noteworthy in this respect. Starting from the body midline and extending to the left or right, participants performed a sequence of the same unimanual action. At some point, a transition occurred from using one hand to using the other. Because the task was performed in only one direction, a possible divergence of the transition point was not considered.

A novel aspect that we introduce in this paper is the aspect of an asymmetry or a preference in the action-selection process. From the perspective of multiple-timescale dynamics, it is interesting to analyze the way in which a long-term bias potentially influences the planning. Within the focus of this study, hand preference is such a long-term bias, known to affect limb selection. This asymmetry arises over a developmental timescale determined by factors that are not yet fully understood (e.g., Hopkins \& Rönnqvist, 1998). Nevertheless its influence cannot be denied, and must be taken into account in any serious model. A fundamental reason why this could be of more general interest for cognitive science is that it gives us a glimpse at how preferences as functional or structural asymmetries are part of the perception-action cycle, at the level of planning in goal-directed behavior. 
Summarizing, the goal of this paper is twofold. First, adding to the empirical realm and theoretical discussion on limb selection the aspect of multiple-timescale dynamics. To investigate this, we performed experiments in which the sequence of earlier limb selections was manipulated, thereby varying its possible effects on subsequent selections. Second, introducing a working dynamical model for the action-selection process underlying limb selection, which integrates and extends the mechanisms proposed by Gabbard et al. (2000; 2004). In this way these mechanisms are treated in accordance with Newell's (1986) view as constraints on action selection rather than as single-cause all-or-none explanations of this kind of behavior. We will show that the model reproduces the results of the reported experiments, and of Gabbard et al.'s (1997) original task.

We report two experiments. In both experiments, adult participants who were sitting at a table had to pick up a small cube on each trial, and displace it to a box in front of them. In Experiment 1, participants received a series of trials in which the cubes were first placed at lateral positions, either four times on the extreme left side or four times on the extreme right side. This constituted a series of memory trials, building a memory trace (i.e., a short-term bias) for using one particular hand. To measure the possible effect of this memory trace on subsequent hand choices, this set of memory trials was followed by two more trials in which the object was presented on the participant's midline. For each participant, a set of memory trials was offered to the preferred hand and non-preferred hand, in two separate conditions. In Experiment 2, right-handed participants received a clockwise and counter-clockwise sequence of cube presentations on the nine different positions in Gabbard et al.'s (1997) task. Each participant received both sequences in two separate conditions. The main objective in this experiment was to detect differences in the pattern of hand use across hemispace as a function of the type of sequence that has been performed. 
In light of the results found by Gabbard et al. (1997) and our view on perseveration, we formulated two major hypotheses with respect to Experiment 1. First, we expected an increase in the number of non-preferred-hand uses at midline after a set of memory trials at the nonpreferred hand. This short-term memory effect is what we call perseverative limb selection. A small increase in the number of preferred-hand uses was expected after a set of memory trials at the preferred hand, due to a ceiling effect. Second, because of the difference in handedness strength between the two laterality groups, we expected to find a difference in the size of the perseveration effect. Left-handers were hypothesized to demonstrate a stronger effect of perseveration, because they already switch more easily to their non-preferred hand. Therefore, the effect of the short-term bias on future choices was reasoned to stand a better 'chance' in the process next to the weaker (compared to right-handers) long-term bias of the hand preference. In Experiment 2, when presenting the cubes in a clockwise sequence compared to the random task, we expected to see a (spatial) delay in the location of the switch from grasping the cube with the left hand to grasping it with the right hand. The same delay was expected for right-hand grasping switching into left-hand grasping in the counter-clockwise condition.

\section{EXPERIMENT 1}

Method

Participants. Twenty strongly left-handed adults (5 male and 15 female) and 24 strongly right-handed adults (4 male and 20 female). The direction and strength of the handpreference was assessed using the four items of the manual part of the Lateral Preference Inventory for measurement of handedness, footedness, eyedness, and earedness (Coren, 1993). As was expected, it was more difficult to find strongly left-handers (i.e., with four consistent left-hand answers on the inventory) than strongly right-handers. The mean age of 
the participants was 21.8 years (range: 19 - 28 years). None of the participants had a visual or physical impairment that could interfere with task performance. In particular, they all had normal or corrected to normal eyesight.

Procedure. Participants were seated on a chair in front of a table $(75 \mathrm{~cm}$ x $150 \mathrm{~cm} \times 75$ cm high), with an experimenter facing them on the opposite side. On this table nine locations were marked on a half circle, with the participant at its center, at slightly less than arms length away. These locations were set at 20 degrees intervals, starting at 10 degrees relative to the edge of the table. This composed the following series: $10^{\circ}, 30^{\circ}, 50^{\circ}$, and $70^{\circ}$ on the left side of the participant, $90^{\circ}$ (equaling the participant's midline), and $-70^{\circ},-50^{\circ},-30^{\circ}$, and $-10^{\circ}$ on the right side of the participant (see Figure 1). In the following, the minus sign will be used to denote locations in the right hemispace.

A 1-inch ${ }^{3}$ cube was placed at one of these locations on each trial. Participants were asked to grasp the cube with one hand and transport it to a small box in front of them. Before the start of each trial, participants were instructed to close their eyes while the experimenter positioned the cube. After a verbal signal ("OK") from the experimenter, the participant opened the eyes and reached for the object. It was made clear that there was no need for a speedy reaction, but that enough time could be taken to make a decision to use one of the hands. Participants were unaware of the goal of the experiment.

The experiment consisted of three conditions. Two conditions started by establishing a short-terms bias for one of the hands, by offering a set of four memory trials: $M_{1}, M_{2}, M_{3}$ and M4. In one of the conditions the participant's left hand was trained, in the other condition the participant's right hand. Note that this means that each participant was trained on the preferred hand (PHM) as well as on the non-preferred hand (NPHM). In the memory trials, the cube was presented successively at the $10^{\circ}, 30^{\circ}, 10^{\circ}$, and $30^{\circ}$ locations on the left side (left-hand memory), or at the $-10^{\circ},-30^{\circ},-10^{\circ}$, and $-30^{\circ}$ locations on the right side (right-hand 
memory). Participants were free to choose the hand to grasp the cube with. In two subsequent 'neutral' trials, $\mathrm{N}_{1}$ and $\mathrm{N}_{2}$, the cube was presented at midline $\left(90^{\circ}\right)$, allowing us to determine the participant's choice of limb on midline after the set of memory trials. The order of the two memory conditions was counterbalanced.

In between these two memory conditions, Gabbard et al.’s (1997) experiment was performed as a third condition. The rationale for this was that this enabled us to establish a baseline proportion of preferred-hand use on midline, in the absence of a specific memory trace. In addition, it enabled us to replicate their findings. This random condition (RC) consisted of a series of nine trials in which the cube was placed on the table in each of the nine locations of Figure 1 in a completely randomized order. The set up of the experiment closely followed the original procedure by Gabbard et al.

\section{Results and discussion}

Random condition. In the random condition we found a similar pattern of hand use as Gabbard et al. (1997). Despite this overall pattern, we found no significant differences between left-handers and right-handers on any of the specific locations. This may be due to a relatively small number of participants, which was about one-third of Gabbard et al.

Memory conditions. The following results include 19 left-handed participants and 17 right-handed participants. The reason for these numbers to be smaller than the total number of participants in each handedness group, is that not all participants turned out to be 'trainable' on their non-preferred hand. In our experimental procedure we deliberately did not instruct the participants to use the ipsilateral hand in the memory trials, because of the obvious possibility of transfer of this instruction to the neutral trials. As a result, a number of participants used their preferred hand rather than their non-preferred hand when the cubes were presented on their non-preferred side (i.e., contralateral with respect to cube position), 
despite the awkward across-midline reaching that this entails. As might be expected from the well-known difference in laterality strength between the handedness groups (although not found here), this number was considerably larger in the group of right-handers (seven participants) than in the group of left-handers (one participant). The participants who were not 'trainable' all used their preferred hand at all six trials in both conditions.

In the following analyses, only the data of the participants who completed all memory trials $\left(\mathrm{M}_{1}\right.$ to $\left.\mathrm{M}_{4}\right)$ with the appropriate hand were used, that is, ipsilateral with respect to the object. This constituted a group with a well-established short-term bias for that hand, and for whom perseverative limb selection might be expected. The results of the two memory conditions are presented in Table 2. From the replication of Gabbard et al.'s (1997) experiment in the random condition, we obtained a baseline proportion of preferred-hand use on midline $\left(90^{\circ}\right)$. This was .84 for the left-handers and .88 for the right-handers. In the following we will discuss the main findings of the experiment.

Table 2. Results of the memory conditions of Experiment 1: Proportion of preferred-hand use in the last two memory trials $\left(M_{3}\right.$ and $\left.M_{4}\right)$ and the two neutral trials $\left(N_{1}\right.$ and $\left.N_{2}\right)$. The proportion of preferred-hand use for the $90^{\circ}$ location in the random task (Experiment 1) was .84 for the lefthanders, and .88 for the right-handers.

\begin{tabular}{ccccc}
\hline Group & $\mathrm{M}_{3}$ & $\mathrm{M}_{4}$ & $\mathrm{~N}_{1}$ & $\mathrm{~N}_{2}$ \\
\hline $\begin{array}{c}\text { Left-handers }(N=19) \\
\text { Preferred-hand memory }\end{array}$ & 1.00 & 1.00 & 1.00 & 1.00 \\
$\quad$ Non-preferred-hand memory & 0.00 & 0.00 & .53 & .58 \\
\hline $\begin{array}{l}\text { Right-handers }(N=17) \\
\text { Preferred-hand memory }\end{array}$ & 1.00 & 1.00 & .94 & .88 \\
Non-preferred-hand memory & 0.00 & 0.00 & .53 & .65 \\
\hline
\end{tabular}


First, the data of the two handedness groups were collapsed and analyzed as a single group of 36 participants. We tested if the proportions of preferred-hand use on midline differed between the two memory conditions (PHM and NPHM) and the random condition (RC). This was done for the first neutral trial $\left(\mathrm{N}_{1}\right)$ and the second neutral trial $\left(\mathrm{N}_{2}\right)$ separately, using Cochran tests for comparing three related samples. For the $\mathrm{N}_{1}$ trial there was a significant difference between PHM, NPHM and RC, $Q(d f=2, N=36)=26.000, p<.001$. This was also the case for the $\mathrm{N}_{2}$ trial, $Q(d f=2, N=36)=18.000, p<.001$. These analyses show that, overall, the participants demonstrated a different pattern of limb selection on midline, depending on the sequence of earlier choices that have been made. This clearly shows that perseveration is present in adult limb selection, at least in unimanual grasping on midline.

To analyze these findings more closely, we used McNemar tests for comparing two related samples to test whether the differences in the proportions of preferred-hand use in the combinations PHM - RC, NPHM - RC, and PHM - NPHM were significant. For both trials $\left(\mathrm{N}_{1}\right.$ and $\left.\mathrm{N}_{2}\right)$ there were significant differences in the proportions of preferred-hand use between NPHM and RC ( $p<.001$, and, $p<.001$, respectively), and between PHM and NPHM ( $p<.01$, and, $\mathrm{p}<.001$, respectively). After performing a set of memory trials with the non-preferred hand, participants subsequently used this hand more often, compared to performing a set of memory trials with the preferred hand or after having no specific memory trace at all. This is still true for the second subsequent $\mathrm{N}_{2}$ trial. Taken together, these results confirm the conclusion of perseverative limb selection in adult unimanual grasping.

Next, to study this effect as a function of handedness, we analyzed the results for lefthanders and right-handers separately. For both handedness groups, Cochran tests revealed that the proportions of preferred-hand use on midline significantly differed between PHM, NPHM 
and $\mathrm{RC}$ in both neutral trials (all $p$ 's $<.01$, except for the $\mathrm{N}_{2}$ trial for the right-handers, $p<$ $.05)$.

Finally, McNemar tests revealed that for both handedness groups, the differences in the proportions of preferred-hand use were significant between NPHM and RC $(p<.05$, for lefthanders and right-handers), and between PHM and NPHM ( $p<.01$ and $\mathrm{p}<.05$, for lefthanders and right-handers respectively), for the first neutral trial. For the second neutral trial, only the difference between PHM and NPHM was significant in the left-handed group $(p<$ .01). Although perseverance was present for both handedness groups in the first neutral trial, these analyses demonstrate a difference in the strength of the effect for the second neutral trial. Right-handers no longer use the non-preferred hand in the second subsequent trial more often after a specific memory trace of that hand. In other words, the effect of the earlier limb selections is no longer strong enough to overrule the hand preference.

\section{EXPERIMENT 2}

\section{Method}

Participants. Fifteen strongly right-handed adult volunteers (3 male and 12 female) with a mean age of 21.4 years (range: 19 - 25 years) participated in the experiment.

Procedure. The general procedure was the same as in the previous experiment and closely followed the original procedure by Gabbard et al. (1997). Experiment 2 consisted of three conditions, each with a series of nine successive cube presentations. Two of them where sequential conditions: a clockwise condition and a counter-clockwise condition. In the clockwise condition, starting with the $10^{\circ}$ location on the participant's left side, the experimenter sequentially placed the cube on each following location of Figure 1, up until the $-10^{\circ}$ location on the participant's right side. In the counter-clockwise condition the experimenter placed the cube in a similar but opposite fashion, starting on the participant's 
right side at the $-10^{\circ}$ location. The order of the two sequential conditions was counterbalanced. As in Experiment 1, Gabbard et al.'s experiment was replicated as a third condition in between the two sequential conditions. In this condition the cube was randomly placed in each of the nine locations of Figure 1.

\section{Results and discussion}

The results of the two sequential conditions are presented in Table 3. To highlight the effects, the proportions of preferred-hand use for the $50^{\circ}, 70^{\circ}$, and $90^{\circ}$ locations are also shown in Figure 2. Figure 2 also shows the scores of the participants for these trials in the random condition.

As shown in Figure 2, a spatial delay in the location of the switch from preferred-hand use to non-preferred-hand use is present between the two sequential conditions, and between the sequential conditions and the random condition. The occurrence of this effect at the level of individual participants was as follows: 67\% (10 participants) displayed a hysteresis effect, whereas $33 \%$ (5 participants) had a critical boundary in their selection of limb. This result reveals the presence of hysteresis in adults' selection of limb for unimanual grasping, identifying it as a nonlinear dynamical system.

Table 3. Results of the sequential conditions of Experiment 2: Proportion of preferred-hand use for the nine (sequentially presented) object positions.

\begin{tabular}{lccccccccc}
\hline \multicolumn{1}{c}{ Sequence } & \multicolumn{4}{c}{ Left hemispace } & \multicolumn{4}{c}{ Right hemispace } \\
\multicolumn{1}{c}{$(N=15)$} & $10^{\circ}$ & $30^{\circ}$ & $50^{\circ}$ & $70^{\circ}$ & $90^{\circ}$ & $-70^{\circ}$ & $-50^{\circ}$ & $-30^{\circ}$ & $-10^{\circ}$ \\
\hline Clockwise & .13 & .07 & .13 & .20 & .73 & 1.00 & 1.00 & 1.00 & 1.00 \\
Counter-Clockwise & .07 & .07 & .47 & .67 & 1.00 & 1.00 & 1.00 & 1.00 & 1.00 \\
\hline
\end{tabular}




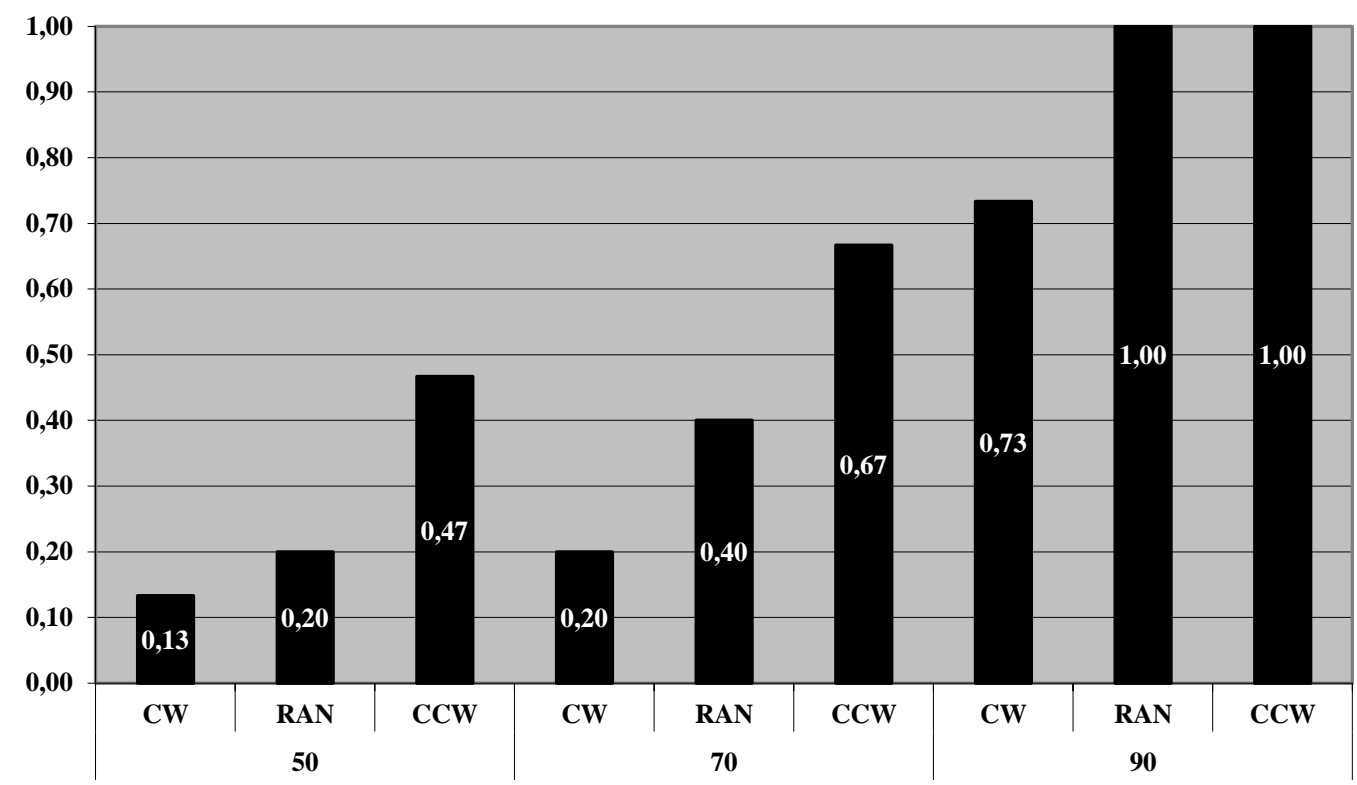

Figure 2. Results of Experiment 2: Presented are the proportions of preferred-hand use for the $50^{\circ}$, $70^{\circ}$, and $90^{\circ}$ locations for the clockwise sequence, the counter-clockwise sequence and the random sequence.

For the participants who showed hysteresis, the average size of the spatial delay was 1.3 locations, with a standard deviation of 0.48 locations. In the critical-boundary group, one participant used her preferred hand consistently at all nine cube location in all three conditions. Another participant of this group used her preferred hand up to the $50^{\circ}$ location in all three conditions. The other three participants demonstrated an equal pattern of limb selection; all three switched to the non-preferred hand at the $70^{\circ}$ location in all three conditions.

\section{DYNAMIC MODEL}

To reproduce the effects of perseveration and hysteresis in limb selection, we propose a model based on a dynamical action-selection process. The model will have to explain how the choice to use a particular hand for grasping the cube emerges as the resulting behavior at the 
overt level, from the various external and internal factors that are seem to interact at the covert level of the action-selection process. To accomplish this, the model will be based on a thorough analysis of the tasks, as well as on the relevant features of action planning as discussed in the introduction. We will describe the model's basic features below. This will be followed by a simulation of Experiment 1 and Experiment 2. Finally, we will present an extension of the model, by suggesting a concise implementation of the difference in handedness strength between left-handers and right-handers. This enabled us to reproduce Gabbard et al.'s (1997) findings, in particular replicating the differences in the patterns of hand use between the two handedness groups.

The model we introduce is closely related to the general category of dynamic field models. This type of models has been used to describe various kinds of cognitive, goaldirected and automatic human behavior, such as saccadic eye movements and arm movements (Erlhagen \& Schöner, 2002; Kopecz \& Schöner, 1995; Schöner, Kopecz \& Erlhagen, 1997), perseverative reaching movements (Thelen et al., 2001), spatial memory (Schutte \& Spencer, 2002; Schutte, Spencer \& Schöner, 2003; Spencer \& Schöner, 1998), and recently the habituation paradigm (i.e., "violation of expectancy" and "preferential looking") has been added to this list (Schöner \& Thelen, 2003; Thelen \& Whitmyer II, 2004). The success of this type of models lies in its generality; it can be used for any number of perceptual-motor tasks without altering its basic assumptions.

Although not constructing a field-theoretical model, we will use two of its main concepts for building a model that will simulate the mapping between the continuous spatial (and temporal) aspects of the cube presentation and the discrete choice to use one of the hands for grasping that cube. These concepts are (see Erlhagen \& Schöner, 2002): First, the choice to use one of the hands evolves gradually over time and is governed by a continuous 
dynamics. Second, this governing dynamics receives multiple inputs which integrate in the action-selection process.

\section{Model description}

The central feature of the model is the activation levels of the action-selection process for each of the two hands. In principle, the planning process for reaching to a certain location has to be viewed as a continuously distributed field of activation (Erlhagen \& Schöner, 2002; Thelen et al., 2001). In this paper, however, we are not interested in the direction of the reach, but in the hand chosen to execute the reach with. Accordingly, we will only follow two sites, each representing the action-selection process to use one of the hands (cf. Schöner \& Thelen, 2003). The sites will be assigned activation functions $u_{L}(t)$ and $u_{R}(t)$, for the left hand and right hand respectively. The choice to use a particular hand is determined by the activation level of the corresponding site. The likelihood of this choice is closely related to the numerical value of the site's activation functions at a particular instant in time, or rather to the relative proportion of both activation levels. When the activation level passes a certain threshold, a motor plan for using the corresponding hand will be executed, which results in the actual onset of a reaching movement of the corresponding limb. The threshold levels for the left-hand site and right-hand site are the same. When the activation levels of both sites pass the threshold simultaneously, or within a certain time window, this will be interpreted as bimanual behavior.

The sites are mutually coupled by cross-lateral inhibitory connection. This means that when the activation level of one site increases (i.e., the likelihood of using the corresponding hand grows), this has a decreasing effect on the activation level of the other site (i.e., the likelihood of that hand being used shrinks). The key feature we introduce in this model is that the process is inherently laterally biased, which is determined by unequal connection strengths (cf. Thelen \& Whitmyer II, 2004). The strength of the inhibition will, in general, be 
different for both sites, symbolized by the numerical values of the parameters $c_{L}$ and $c_{R}$. Essential to the model's ontology and its behavior is that a difference in inhibition strength (i.e., the difference in the numerical values of the $c$ parameters) expresses a difference in hand preference or laterality. The more consistent the hand preference, that is, the more one hand is favored above the other, the larger the difference in the inhibition strength. Note that, in the dynamics of the model, this difference will only lead to an increased likelihood for using one hand over the other, not to a static preference to use one hand irrespective of external or internal stimulation. The functional form of the inhibitory connections is that of a sigmoid shape:

$$
\sigma(v)=\frac{1}{1+e^{-\beta\left(u(t)-u_{0}\right)}},
$$

where the slope parameter $\beta$ expresses the steepness of the function and $u_{0}$ is a threshold. The function $u(t)$ is the activation function of one of the two sites, which drives the inhibition.

In general, limb selection will depend on the spatial layout of external stimulation, that is, the locations of relevant objects with respect to the actor's body. The relation between external stimulation and corresponding manual behavior obeys some very robust laws. For example, the effect of spatial compatibility in stimulus-response activity for hand use, which is related to the more general Simon effect (Simon, 1969) and Poffenberger effect (Poffenberger, 1912). To enable the model to exhibit such law-like behavior, the sites will receive (perceptual) input according to the lateral arrangement of the external stimulation. The input for both sites will not have equal strength, but will be closely related to the cube's location in each trial. As a result of this, changes in the likelihood to use a particular hand will arise. The strengths of the perceptual inputs to the two sites, as a function of the cube's 
location with respect to the participant, are presented in Figure 3 for both the left-hand site and right-hand site. In the model, the perceptual (per) input will be symbolized by $I^{p e r}{ }_{L}(t)$ and

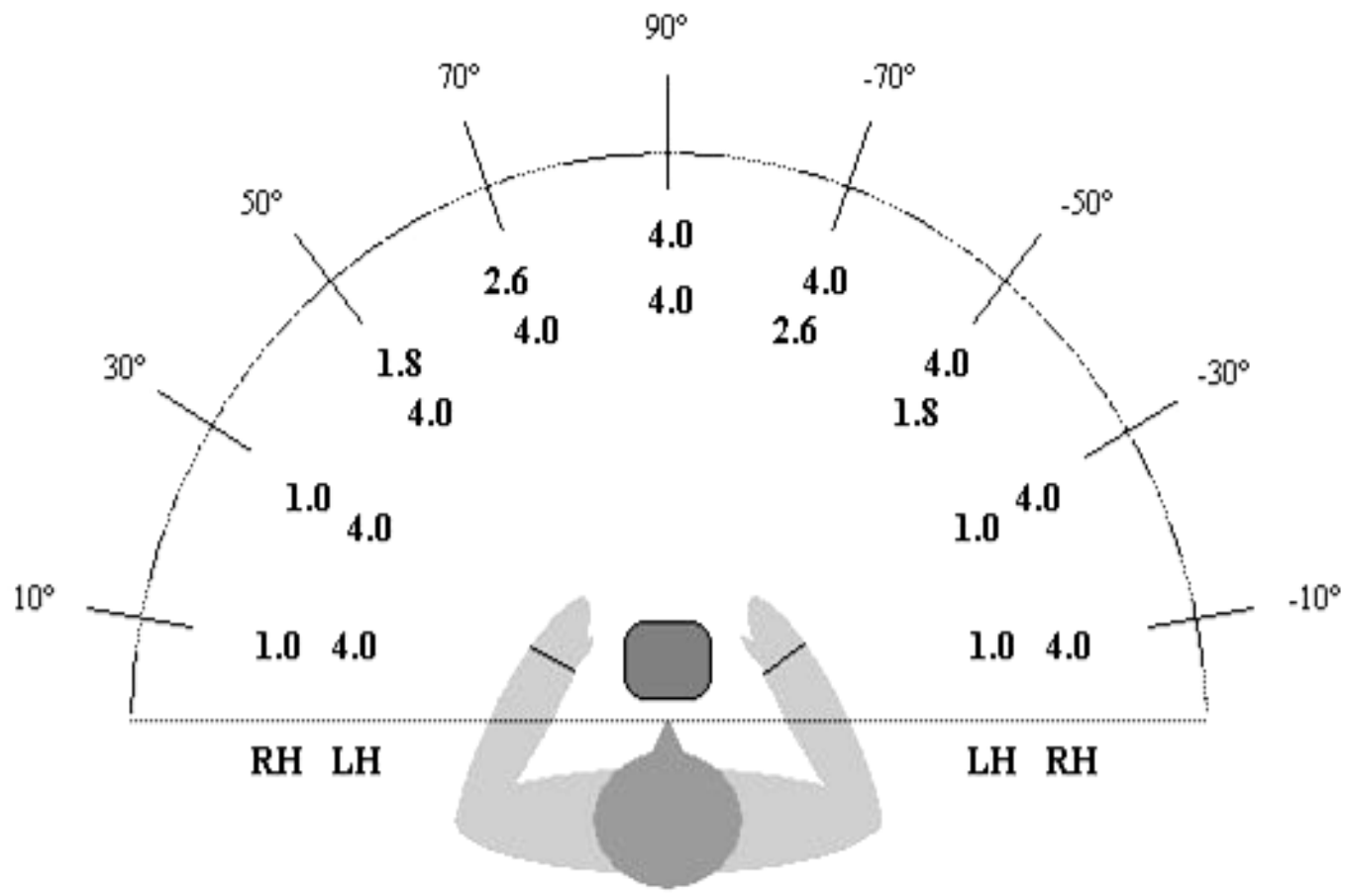

$I_{R}^{p e r}(t)$, for the left hand and right hand, respectively.

Figure 3. Strength of the perceptual input for the left hand (LH) and right hand (RH) for the different object locations.

Critical to the phenomena of perseveration and hysteresis is that making choices for actions to take is a process that works on multiple timescales. To understand the results of both Experiment 1 and Experiment 2, we have to realize that the choices to use one of the hands in a particular trial, among other things, depends on the hand choices in earlier trials. Therefore, in addition to the perceptual input, a second input source flows into the actionselection process. This input is internal to the action system (i.e., organismic; Newell, 1986), and originates from the (motor)memory of limb selection in earlier trials. In the model, this memory (mem) input will be symbolized by $I^{m e m}(t)$ and $I_{R}^{m e m}(t)$, for the left hand and right hand, respectively. 
Starting from zero at the beginning of each condition in the experiment, this input source builds up or breaks down for each hand, depending on the particular pattern of limb selections over successive trials. Every time a particular hand is used, that is, the activation function of a particular site has passed the threshold, the strength of the memory input for that site is increased with 1.2 before the next trial. When a hand is not used in a trial the strength of the memory input will decrease to the zero level. Combining the perceptual source and memory source of input, the total input in the model now has the following form:

$$
\begin{aligned}
& I_{L}(t)=I_{L}^{\text {per }}(t)+I_{L}^{\text {mem }}(t) ; \\
& I_{R}(t)=I_{R}^{\text {per }}(t)+I_{R}^{\text {mem }}(t),
\end{aligned}
$$

for the left-hand site and the right-hand site, respectively.

In the absence of input, the activation functions will decay, eventually returning to a resting level $h$. This resting level establishes a basic activation level, which corresponds to an a-priori willingness or likelihood to use the corresponding hand in a stimulus-free or stimulusneutral environment. The resting level $h$ will have an equal value for both sites.

The final part of the dynamics is a noise factor $\xi(t)$, which is supposed to be Gaussian white noise. Noise is common in (realistic models of) behavioral, neural and biological systems (e.g., Mainen \& Sejnowski, 1995; Schöner, Haken, \& Kelso, 1986; Shinbrot \& Muzzio, 2001; Thelen, Schöner, Scheier \& Smith, 2001). It represents the random fluctuations in the system, which can have a functional significance for the behavior. In particular these can lead to spontaneous reaches with any of the two hands or both.

In sum, the sites obey a continuous dynamics described by a set of two coupled, firstorder nonlinear differential equations. These equations characterize the internal dynamics of 
the action-selection process and integrate the properties described above. In terms of the two equations, the model has the following form:

$$
\begin{aligned}
& \tau \cdot \dot{u}_{L}(t)=-u_{L}(t)+h-c_{L} \cdot \sigma\left(u_{R}\right)+I_{L}(t)+n \cdot \xi(t) \\
& \tau \cdot \dot{u}_{R}(t)=-u_{R}(t)+h-c_{R} \cdot \sigma\left(u_{L}\right)+I_{R}(t)+n \cdot \xi(t) .
\end{aligned}
$$

The 'dot' on the activation functions $u_{L}$ and $u_{R}$ on the left-hand side of the equations represents the derivative with respect to time (i.e., $d u_{L} / d t$ and $d u_{R} / d t$ respectively). As mentioned earlier, a fundamental part of the model's behavior is the decaying property it possesses with time constant $\tau$. This property is expressed by the first term on the right side of each equation.

\section{Simulation details}

All simulations were performed in Matlab (version 6.1, The MathWorks Inc.) on a standard PC. The model equations were integrated using the Euler procedure with 200 time steps of $5 \mathrm{~ms}$ size. This means that single-trial simulations of the model ran for a time period that was comparable to the average duration of making a single hand choice in the experiments (i.e., from perceiving the cube to instantiating the reaching movement). Moreover, this was long enough to assure that the model converged to a highest activation level for a single site, which reflects the experimental procedure where participants were given ample time to decide which hand to use. Although the integration procedure equals exactly one second of the selection process, at this point the model makes no specific statements yet about the exact timing of this process.

All simulations are based on 500 runs of the model. These repetitions are necessary to assure convergence of the model and because this way the noise term enabled us to obtain a 
clear distribution in the results (statistics). A complete experimental procedure was simulated for each fictive participant (run) in all simulations presented below. The strengths of the perceptual input and memory input were set before the start of every trial. After every trial, the strength of the memory input was updated. Both inputs were present during the entire within-trial simulation, that is, all 200 time steps for each trial.

\section{Parameters settings}

First, the model was used to simulate Experiment 1 and Experiment 2. In order to get the closest fit as possible with the results of both experiments, the following numerical values for the model parameters were used: The strengths of the cross-lateral inhibition terms were set to the values $c_{P}=1.8$ for the preferred hand and $c_{N P}=5.1$ for the non-preferred hand. The steepness $\beta$ of the sigmoid curve in the cross-lateral inhibition was set to 0.05 , and the threshold $v_{0}$ to 0 . The resting level $h$ received a value of -0.8 . The strength $n$ of the noise was set to 0.8 . The time constant $\tau$ was set to a value of 3 . This parameter setting was fixed for the

simulations of both experiments. Consequently, the only difference between the simulations is the order, locations and number of cube presentations, and the corresponding perceptual input and memory input build up this entails. Fundamentally, the model's behavior, therefore, has to account for the empirical effects of perseveration and hysteresis on the basis of this variation in input dynamics and its internal dynamics. After this Gabbard et al.'s (1997) experiment is also simulated to reproduce their results. For this we need a small but reasonable change in the parameter setting.

\section{Simulation of Experiment 1}

The strengths of the perceptual input were set to the values corresponding to the $(-) 10^{\circ}$, $(-) 30^{\circ}$, and $90^{\circ}$ cube locations, as displayed in Figure 3. So, over the subsequent trials $\mathrm{M}_{1} \rightarrow$ 
$\mathrm{M}_{2} \rightarrow \mathrm{M}_{3} \rightarrow \mathrm{M}_{4} \rightarrow \mathrm{N}_{1} \rightarrow \mathrm{N}_{2}$ in each of the two memory conditions of Experiment 1, the perceptual input for each of the two sites was set to the following numerical values: $4.0 \rightarrow 4.0$ $\rightarrow 4.0 \rightarrow 4.0 \rightarrow 4.0 \rightarrow 4.0$ for the site corresponding to the hand ipsilateral to the object, and $1.0 \rightarrow 1.0 \rightarrow 1.0 \rightarrow 1.0 \rightarrow 4.0 \rightarrow 4.0$ for the site corresponding to the hand contralateral to the object.

Table 4 presents the results of the simulations for 500 runs of each of the two memory conditions of Experiment 1. Shown are the last two memory trials and the two neutral trials.

Table 4. Results of 500 runs of the model simulating the memory conditions of Experiment 1: Proportion of preferred-hand use in the last two memory trials $\left(M_{3}\right.$ and $\left.M_{4}\right)$ and the two neutral trials $\left(N_{1}\right.$ and $\left.N_{2}\right)$.

\begin{tabular}{ccccc}
\hline Condition & $\mathrm{M}_{3}$ & $\mathrm{M}_{4}$ & $\mathrm{~N}_{1}$ & $\mathrm{~N}_{2}$ \\
\hline & & & & \\
Preferred-hand memory & 1.00 & 1.00 & 1.00 & 1.00 \\
Non-preferred-hand memory & .03 & .01 & .50 & .64 \\
\hline
\end{tabular}

Figure 4 presents some typical results of single-participant simulation of the memory conditions. Figure 4a shows a participant who received a set of four memory trials at the nonpreferred hand. As shown in the plots of the $\mathrm{N}_{1}$ trial and the $\mathrm{N}_{2}$ trial, this fictive participant kept on using the non-preferred hand after having been trained at this hand in trials $\mathrm{M}_{1}$ to $\mathrm{M}_{4}$. Figure $4 \mathrm{~b}$ presents a participant, also trained at the non-preferred hand, but demonstrating no perseveration. In the $\mathrm{N}_{1}$ trial and the $\mathrm{N}_{2}$ trial, this fictive participant switches to using the preferred hand, despite having used the non-preferred hand four times in trials $\mathrm{M}_{1}$ to $\mathrm{M}_{4}$. In terms of the model's dynamics, this behavior results from the dynamical interplay between the perceptual input (on-line bias), memory input (short-term bias) and the preference (longterms bias). In the two neutral trials, when perceptual input has equal strength for both sites 
a)
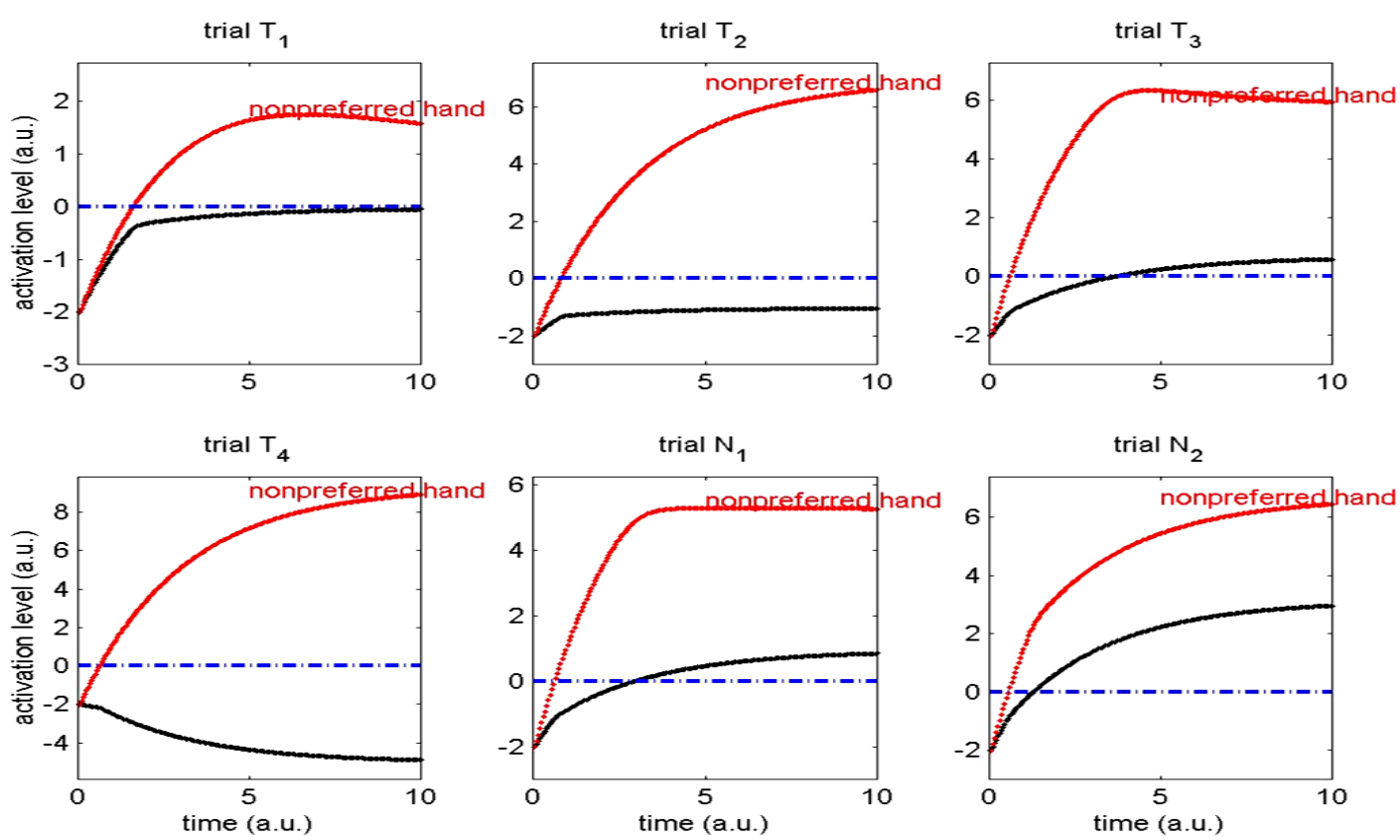

b)
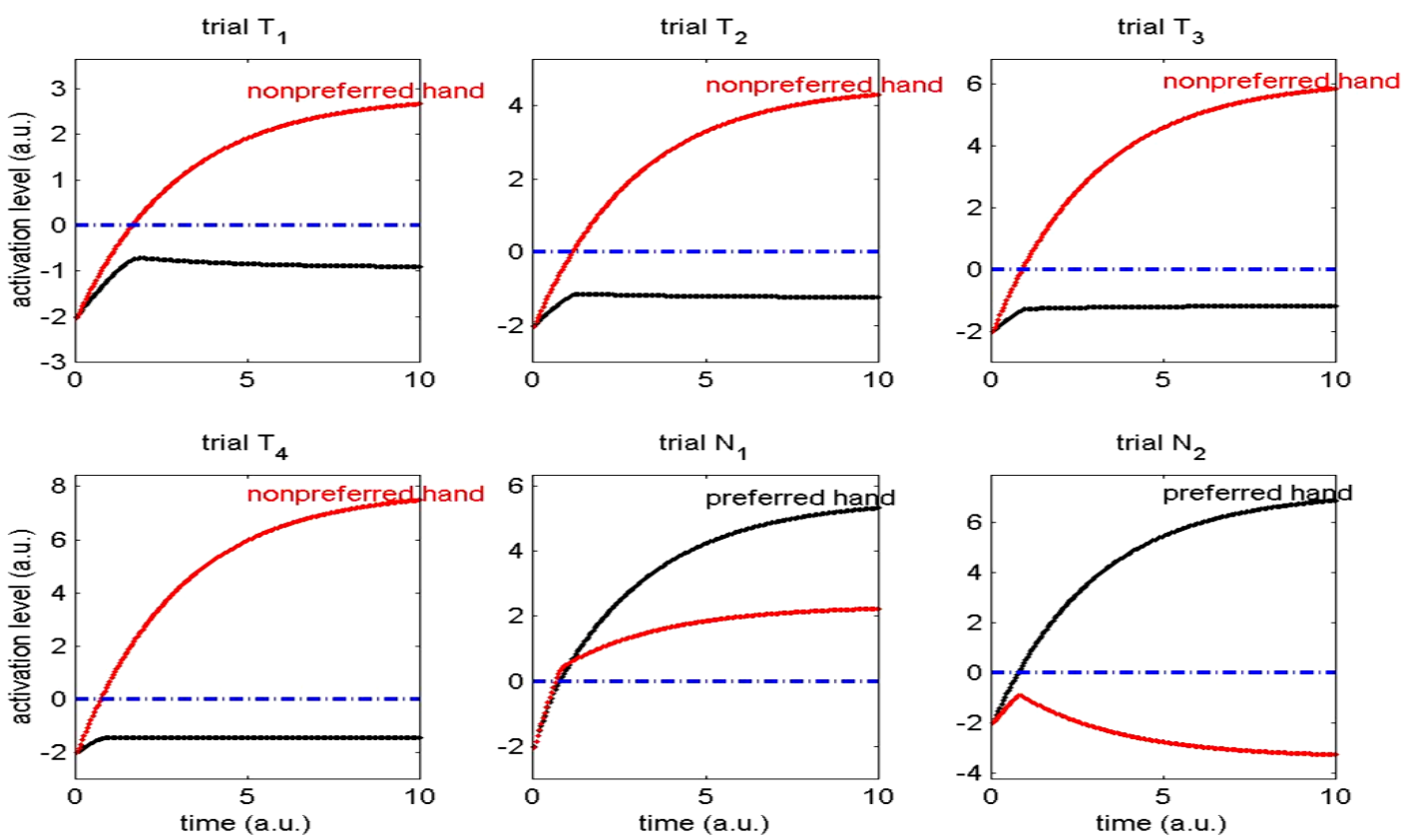

Figure 4. Examples of a single-subject simulation of the memory conditıons of Experiment 1 . I ne dotted line at the zero-level activation denotes the point at which the inhibition starts to affect the dynamics of the action-selection process. a) Subject in the non-preferred-hand memory task showing perseveration. b) Subject in the non-preferred-hand memory task showing no perseveration. 
again, the difference in memory strength that has built up during the memory trials was not strong enough to overrule the hand preference.

\section{Simulation of Experiment 2}

Over the subsequent trials $\mathrm{T}_{1} \rightarrow \mathrm{T}_{2} \rightarrow \ldots \rightarrow \mathrm{T}_{8} \rightarrow \mathrm{T}_{9}$ in each of the two sequential conditions of Experiment 2, the perceptual input for each of the two sites was set to the following strength: $1.0 \rightarrow 1.0 \rightarrow 1.8 \rightarrow 2.6 \rightarrow 4.0 \rightarrow 4.0 \rightarrow 4.0 \rightarrow 4.0 \rightarrow 4.0$ for the site corresponding to the hand ipsilateral to the location of the cube, and $4.0 \rightarrow 4.0 \rightarrow 4.0 \rightarrow 4.0$ $\rightarrow 4.0 \rightarrow 2.6 \rightarrow 1.8 \rightarrow 1.0 \rightarrow 1.0$ for the site corresponding to the hand contralateral to the location of the cube. These numerical values correspond to the input strengths for each cube location, as displayed in Figure 3.

Table 5 presents the results of the simulations for 500 runs of each of the two sequential conditions of Experiment 2. Figure 5 presents some typical results of a single-participant simulation of the sequential conditions.

Table 5. Results of 500 runs of the model simulating the sequential conditions of Experiment 2:

Proportion of preferred-hand use for the nine (sequentially presented) object positions.

\begin{tabular}{lccccccccc}
\hline \multicolumn{1}{c}{ Sequence } & \multicolumn{3}{c}{ Left hemispace } & \multicolumn{4}{c}{ Right hemispace } \\
& $10^{\circ}$ & $30^{\circ}$ & $50^{\circ}$ & $70^{\circ}$ & $90^{\circ}$ & $-70^{\circ}$ & $-50^{\circ}$ & $-30^{\circ}$ & $-10^{\circ}$ \\
\hline Clockwise & .10 & .05 & .10 & .24 & .62 & .93 & 1.00 & 1.00 & 1.00 \\
Counter-Clockwise & .08 & .08 & .32 & .62 & .96 & 1.00 & 1.00 & 1.00 & 1.00 \\
\hline
\end{tabular}


a)
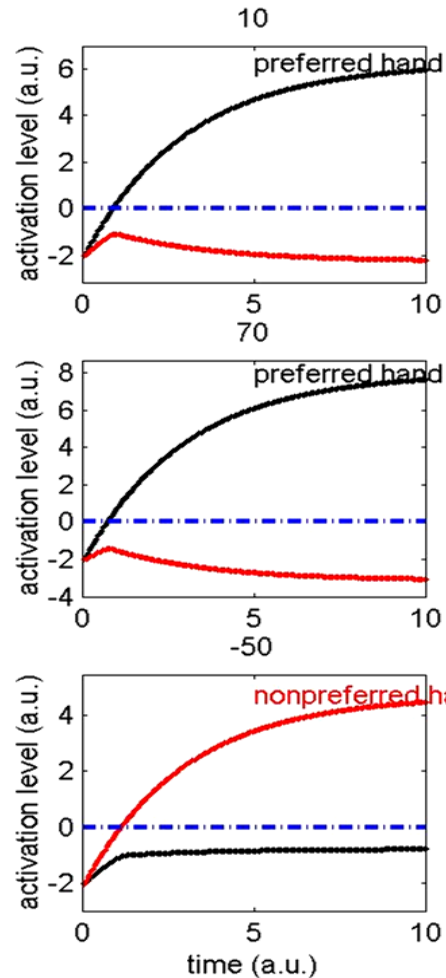

10

b)
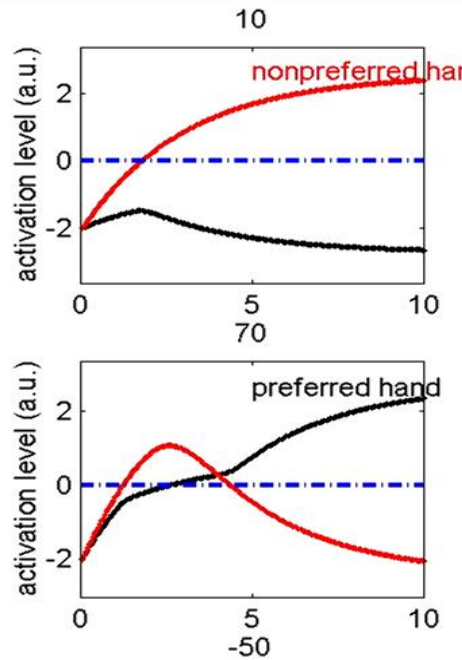

50

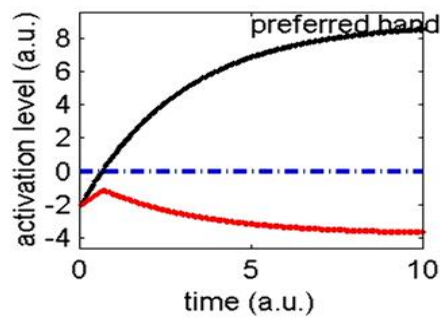

30

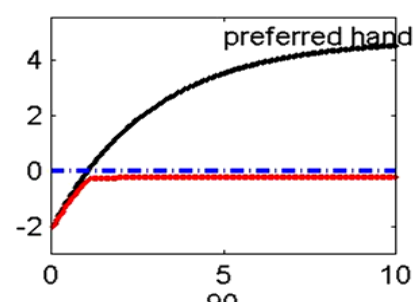

preferredhand
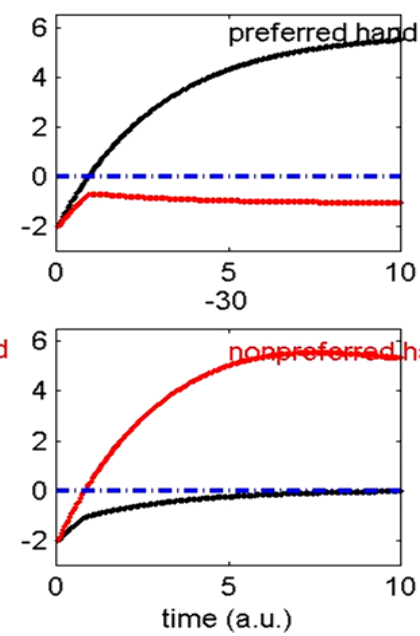

30
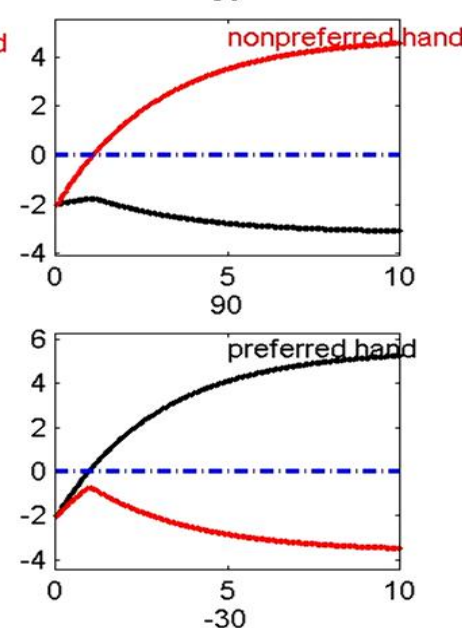

$-30$

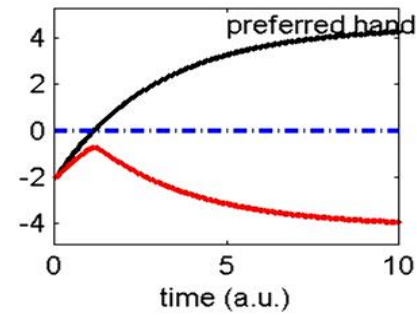

50
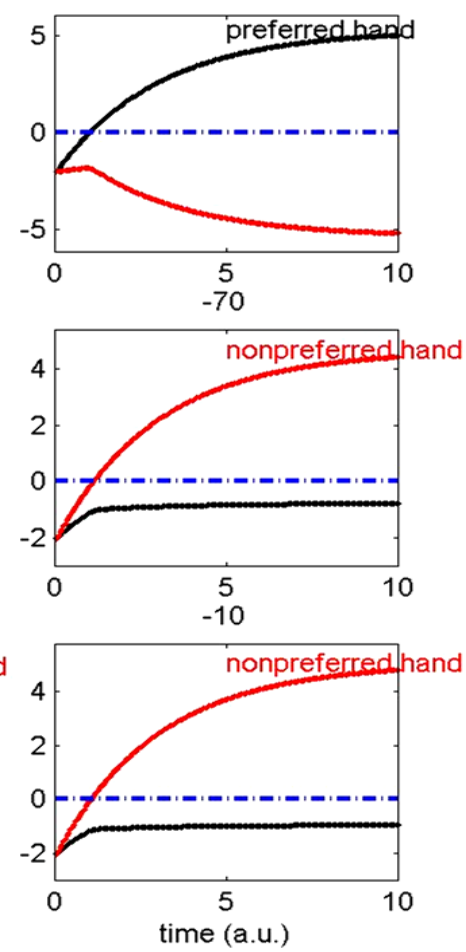

50
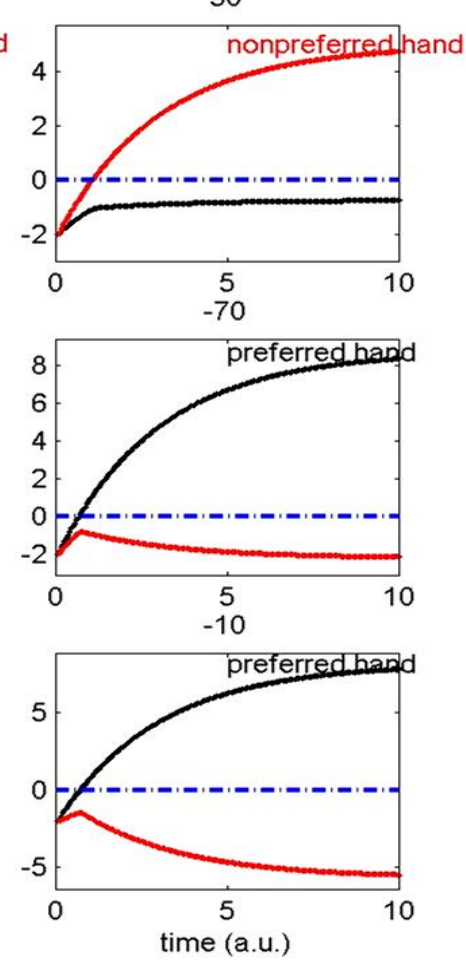

Figure 5. Examples of a single-subject simulation of the sequential conditions of Experiment 2. The dotted line at the zero-level activation denotes the point at which the inhibition starts to affect the dynamics of the action-selection process. a) Subject in the clockwise task showing hysteresis. b) Subject in the counter-clockwise task showing hysteresis. 
Simulation of Gabbard et al. (1997)

One of the main findings of Gabbard et al.'s (1997) experiment was the larger number of (across-midline) grasps into contralateral hemispace (i.e., with respect to the dominant limb) by right-handers compared to left-handers. As mentioned earlier, this was also observed by others. The researchers interpreted this finding as a difference in laterality strength between the two handedness groups. A natural embedding and implementation of this difference in laterality strength in the context of the model presented here, is in terms of unequal cross-lateral inhibition strengths. A larger numerical difference between the two $c$ parameters represents a stronger lateralization, therefore corresponding to right-handers. Similarly, a smaller numerical difference, representing a weaker lateralization, corresponds to left-handers.

In the simulations, the numerical values of the parameters were set as follows: $c_{L}=1.8$ and $c_{R}=3.8$ for left-handed participants, and $c_{L}=5.1$ and $c_{R}=1.8$ for right-handed participants. Except for the cross-lateral inhibition strengths, which we will now treat as characteristic for each handedness group, all other parameters had the same numerical values as in the previous simulations. Simulations were performed for both handedness groups separately. Following the original experimental procedure, the nine locations were simulated in random order on each run.

Table 6 shows the average results over 500 runs of the model simulating the experiment by Gabbard et al. (1997). As can be seen by comparing the simulation results to the experimental results displayed in Table 1, the overall pattern of hand use is similar between the two. 
Table 6. Results of 500 runs of the model simulating Gabbard et al.'s (1997) experiment: Proportion of preferred-hand use for the nine (randomly presented) object positions.

\begin{tabular}{lccccccccc}
\hline \multicolumn{1}{c}{ Group } & \multicolumn{4}{c}{ Left hemispace } & \multicolumn{4}{c}{ Right hemispace } \\
& $10^{\circ}$ & $30^{\circ}$ & $50^{\circ}$ & $70^{\circ}$ & $90^{\circ}$ & $-70^{\circ}$ & $-50^{\circ}$ & $-30^{\circ}$ & $-10^{\circ}$ \\
\hline Left-handers & 1.00 & 1.00 & 1.00 & .98 & .78 & .30 & .10 & .04 & .03 \\
Right-handers & .13 & .09 & .24 & .51 & .93 & 1.00 & 1.00 & 1.00 & 1.00 \\
\hline
\end{tabular}

\section{GENERAL DISCUSSION}

This paper served two main goals: First, we wanted to add to the empirical realm and theoretical discussion on limb selection the aspect of multiple-timescale dynamics, exemplified by the effects of perseveration and hysteresis. In two experiments we manipulated the series of prior hand choices that preceded the selection at some point of a limb for grasping a small object positioned in hemispace. The results of the experiments showed that both perseveration and hysteresis are fundamental in adults' unimanual grasping behavior. Both phenomena are considered as basic for nonlinear multi-timescale dynamical systems. As a result, these experiments revealed that limb selection is governed by an actionselection process that is both dynamical and working on multiple timescales.

Second, we set out to introduce a working dynamical model for limb selection. By integrating and extending earlier proposed mechanisms (Gabbard \& Rabb, 2000), we constructed a dynamical model that brings together various aspects relevant for action selection in goal-directed behavior. The model is capable of letting a single choice, leading to an overt behavioral selection, emerge from various underlying influences. External (i.e., task and environment) constraints as well as internal (i.e., organsimic) constraints are combined in one concise framework, working on at least three different timescales: on-line (perceptual input and the main process itself), short-term (prior choices / system's recent history), and long-term (preferences / functional or structural system characteristics). With the model 
simulations that replicated ours and other experimental results, we demonstrated that a dynamical view of limb selection is not only plausible, but offers a concise and rich framework for further studies in this and adjacent fields.

Below we will discuss some of the model's key aspects and the role they play in the dynamics. Before this however, an important observation regarding the model, which indeed serves as an important criterion for all models, is that there is no one-to-one mapping between parameter settings and effects. Parameter settings alone cannot completely explain the richness of results we were able to reproduce with our simulations. For example, an important set of free parameters is the input strengths to the sites. These of course very much drive the sites' activation levels, and, therefore, to a large extent determine the frequency distributions found in the simulations of Gabbard et al.'s (1997) experiment. Differences in these input strengths between the sites, however, cannot explain the success of the model in replicating the effects of perseveration and hysteresis. Importantly, perseveration and hysteresis were not added to the mathematical implementation of the model as separate elements, as is done in some other models (e.g., Robertson, Guckenheimer, Masnick \& Bacher, 2004). Fundamental to both the experimental effects and the modeling results is the sequential order of the trials in combination with the multi-timescale dynamics of the action-selection process.

The difference in the cross-lateral inhibition strengths of the two sites is primarily responsible for the qualitative difference between the two laterality groups at the behavioral level. Handedness, therefore, is treated as a structural property of the dynamics governing limb selection. Motor memory, in contrast, is treated as an input source that itself builds over an intermediate timescale with respect to the dynamics of the planning process. The dynamic preshaping of the action-selection field by the motor history of the system (memory trace), was already introduced in the dynamic field theory (Erlhagen \& Schöner, 2002; Thelen et al., 2001). Incorporating a structural or functional asymmetry (long-term bias) as an integral part 
of its dynamics, is a novel aspect for this type of models. In this sense the present model is 'more embodied' than the earlier dynamical field model developed for the perseverative reaching behavior.

Related to this is that the concept of cross-lateral inhibition in the action-selection process gives rise to an interesting new perspective on the development of handedness. As is well-known (e.g., Hopkins \& Rönnqvist, 1998; McManus et al., 1988), the consistency of handedness in children grows until at least three years of age. Moreover, from the work of Corbetta and Thelen $(1996 ; 2002)$ we know that the direction of handedness undergoes a series of transitions in the first few months after birth. We hypothesized that the changes in hand preference over age can be modeled by increasing difference between the strengths of the inhibitory connections (Cox \& Smitsman, 2008; Smitsman \& Cox, 2008). 


\section{REFERENCES}

Bryden P. J. (1999). Object and target size effects of manual asymmetries: Is index of difficulty truly a factor. Brain and Cognition, 40, 60-64.

Bryden, P. J., Pryde, K. M., \& Roy, E. A. (1999). Preferential reaching into hemispace: An examination of performance, preference, and task complexity. Brain and Cognition, 40, 64-67.

Bryden, P. J., Pryde, K. M., \& Roy, E. A. (2000). A performance measure of the degree of hand preference. Brain and Cognition, 44, 402-414.

Bryden, M. P., Singh, M., Steenhuisen, R. A., \& Clarkson, K. L. (1994). A behavioral measure of hand preference as opposed to hand skill. Neuropsychologica, 32, 991-999.

Busemeyer, J. R., \& Diederich, A. (2002). Survey of decision field theory. Mathematical Social Sciences, 43(3), 345-370.

Busemeyer, J. R., \& Townsend, J. T. (1993). Decision field theory: A dynamic-cognitive approach to decision making in an uncertain environment. Psychological Review, $100(3), 432-459$.

Calvert, G. A., \& Bishop, D. V. M. (1998). Quantifying hand preference using a behavioral continuum. Laterality, 3, 255-268.

Clark, J. E. (1997). A dynamical systems perspective on the development of complex adaptive skill. In Zukow-Goldring, P. \& Dent-Read, C. (Eds.), Evolving explanations of development: Ecological approaches to organism-environment systems. Washington, D.C.: APA Publications.

Corbetta, D., \& Thelen, E. (1996). The developmental origins of bimanual coordination: A dynamic perspective. Journal of Experimental Psychology: Human Perception and Performance, 22(2), 502-522. 
Corbetta, D., \& Thelen, E. (2002). Behavioral fluctuations and the development of manual asymmetries in infancy: Contributions of the dynamic systems approach. In S. J. Segalowitz \& I. Rapin (Eds.), Handbook of Neuropsychology (Vol. 8, Part I, pp. 309328). Amsterdam, The Netherlands: Elsevier.

Cox, R. F. A., \& Smitsman, A. W. (2006). Action planning in young children's tool use. Developmental Science, 9(6), 629-642.

Cox, R. F. A., \& Smitsman, A. W. (2008). Special section: Towards an embodiment of goals. Theory and Psychology, 18(3), 317-339.

De Agostini, M., Khamis, A. H., Ahui, A. M., \& Dellatolas, G. (1997). Environmental influences in hand preference: An African point of view. Brain and Cognition, 35, 151167.

Deák, G. O., \& Narasimham, G. (2003). Is perseveration caused by inhibition failure? Evidence from preschool children's inferences about word meanings. Journal of Experimental Child Psychology, 86, 194-222.

Deák, G. O., Ray, S. D., \& Brenneman, K. (2003). Children's perseverative appearancereality errors are related to emerging language skills. Child Development, 74, 944-964.

Erlhagen, W., \& Schöner, G. (2002). Dynamic field theory of motor programming. Psychological Review, 109, 547-572.

Fagard, J., \& Dahmen, R. (2003). Cultural influences on the development of lateral preferences: A comparison between French and Tunisian children. Laterality, 9, 67-78.

Farrell, P. S. E. (1999). The hysteresis effect. Human Factors, 41, 226-240.

Gabbard, C., Iteya, M., \& Rabb, C. (1997). A lateralized comparison of handedness and object proximity. Canadian Journal of Experimental Psychology, 51, 176-180.

Gabbard, C., \& Rabb, C. (2000). What determines choice of limb for unimanual reaching movements? Journal of General Psychology, 127, 178-184. 
Gabbard, C., \& Rabb Helbig, C. (2004). What drives children's limb selection for reaching in hemispace? Experimental Brain Research, 156, 325-332.

Grossberg, S. (1980). How does a brain build a cognitive code? Psychological Review, 87, 151.

Haken, H., Kelso, J. A. S., \& Bunz, H. (1985). A theoretical model of phase transitions in human hand movements. Biological Cybernetics, 51, 347-356.

Harris, L. J., \& Carlson, D. F. (1993). Hand preference for visually-directed guiding in human infants and adults. In: J. P. Ward \& W. D. Hopkins (Eds.), Primate laterality: current behavioral evidence of primate asymmetries. New York: Springer.

Hock, H. S., Schöner, G., \& Kelso, J. A. S. (1993). Bistability and hysteresis in the organization of apparent motion patterns. Journal of Experimental Psychology: Human Perception \& Performance, 19, 63-80.

Hopkins, B., \& Rönnqvist, L. (1998). Human handedness: Developmental and evolutionary perspectives. In F. Simion \& G. Butterworth (Eds.), The development of sensory, motor and cognitive capacities in early infancy: From perception to cognition. Psychology Press.

Kelso, J. A. S. (1995). Dynamic patterns: The self-organization of brain and behavior. Cambridge: MIT Press.

Kopecz, K., \& Schöner, G. (1995). Saccadic motor planning by integrating visual information and pre-information on neural dynamic fields. Biological Cybernetics, 73, 49-60.

Leconte, P., \& Fagard, J. (2004). Influence of object spatial location and task complexity on children's use of their preferred hand depending on their handedness consistency. Developmental Psychobiology, 45(2), 51-58.

Mainen, Z. F., \& Sejnowski, T. J. (1995). Reliability of spike timing in neocortical neurons. Science, 268, 1503-1506. 
McManus, I. C., Sik, G., Cole, D. R., Mellon, A. F., Wong, J., \& Kloss, J. (1988). The development of handedness in children. British Journal of Developmental Psychology, $6,257-273$.

Newell, K. M. (1986). Constraints on the development of coordination. In M. G. Wade \& H. T. A. Whiting (Eds.), Motor development in children: Aspects of coordination and control. Dordrecht, The Netherlands: Martinus Nijhoff Publishers.

Newell, K. M. (1989). On task and theory specificity. Journal of Motor Behavior, 21, 92-96. Peters, M. (1990). Phenotype in normal left-handers: An understanding of phenotype is the basis for understanding mechanism and inheritance of handedness. In S. Coren (Ed.), Left-handedness: Behavioral implications and anamolies (pp. 167-192). Amsterdam: North-Holland.

Piaget, J. (1954). The construction of reality in the child. New York: Basic Books.

Poffenberger, A. T. (1912). Reaction time to retinal stimulation with special reference to the time lost in conduction through nervous centers. Arch. Psychology, 23, 1-73.

Robertson, S. S., Guckenheimer, J., Masnick, A. M., \& Bacher, L. F. (2004). The dynamics of infant visual foraging. Developmental Science, 7(2), 194-200.

Schöner, G., Haken, H. \& Kelso, J. A. S. (1986). A stochastic theory of phase transitions in human hand movement. Biological Cybernetics, 53, 442-452.

Schöner, G., \& Kelso, J. A. S. (1988). A synergetic theory of environmentally-specified and learned patterns of movement coordination. I. Relative phase dynamics. Biological Cybernetics, 58, 71-80.

Schöner, G., Kopecz, K., \& Erlhagen, W. (1997). The dynamic neural field theory of motor programming: Arm and eye movements. In P. G. Morassa \& V. Sanguineti (Eds.), Selforganization, computational maps and motor control (Vol. 119, pp. 271-310).

Amsterdam, The Netherlands: Elsevier-North Holland. 
Schöner, G., \& Thelen, E. (2003). Using dynamic field theory to rethink infant habituation. Manuscript submitted for publication.

Schutte, A. R., \& Spencer, J. P. (2002). Generalizing the dynamic field theory of the A-not-B error beyond infancy: Three-year-olds' delay- and experience-dependent location memory biases. Child Development, 73, 377-404.

Schutte, A. R., Spencer, J. P., \& Schöner, G. (2003). Testing the dynamic field theory: Working memory for locations becomes more spatially precise over development. Child Development, 74, 1393-1417.

Shinbrot, T., \& Muzzio, J. (2001). Noise to order. Nature, 410, 251-258.

Simon, J. R. (1969). Reactions toward the source of stimulation. Journal of Experimental Psychology, 81, 174-176.

Smitsman, A. W., \& Cox, R. F. A. (2008). Perseveration in tool use: A window on the dynamics of the action-selection process. Infancy, 13(3), 249-269.

Sophian, C., \& Wellman, H. (1983). Selective information use and perseveration in the search behavior of infants and young children. Journal of Experimental Child Psychology, 35, 369-390.

Spencer, J.P., \& Schöner, G. (2000). A dynamic field model of location memory. Paper in the Proceedings of the 22nd Annual Conference of the Cognitive Science Society, Philadelphia, PA.

Steenhuisen, R. E., \& Bryden, M. P. (1999). The relation between hand preference and hand performance: What you get depends on what you measure. Laterality, 4, 3-26.

Thelen, E., \& Smith, L. B. (1994). A dynamic systems approach to the development of cognition and action. Cambridge, MA: MIT Press.

Thelen, E., Schöner, G., Scheier, C., \& Smith, L. B. (2001). The dynamics of embodiment: A field theory of infant perseverative reaching. Behavioral and Brain Sciences, 24, 1-86. 
Thelen, E., \& Whitmyer II, V. (2004). Using dynamic field theory to conceptualize the interface of perception, cognition and action. Manuscript submitted for publication.

Tuller, B., Case, P., Ding, M., \& Kelso, J. A. S. (1994). The nonlinear dynamics of speech categorization. Journal of Experimental Psychology: Human Perception and Performance, 20, 3-16.

Zelazo, P. D., \& Reznick, S. J. (1991). Age-related asynchrony of knowledge and action. Child Development, 62, 719-735. 